\title{
CONDICIONES PARA LA INNOVACIÓN, CULTURA ORGANIZACIONAL Y SOSTENIBILIDAD DE LAS ORGANIZACIONES*
}

\author{
Recibido: 2 de agosto de 2014 Aprobado: 18 de agosto 2015 \\ Néstor Sanabria** \\ Julio César Acosta-Prado*** \\ Gustavo Rodríguez Albor ${ }^{* * *}$
}

\section{RESUMEN}

Las organizaciones se pueden asumir como el resultado de las necesidades históricas del entorno y de los sistemas sociales en su proceso evolutivo, y su sostenibilidad depende de la capacidad de entender su propia complejidad. Este artículo propone que la dotación de condiciones para la innovación, como expresión de la cultura organizacional, es una opción garante de sostenibilidad y requiere de un proceso no uniforme, ni predecible, es decir, un proceso complejo. Las reflexiones apuntan a que las empresas, para garantizar su sostenibilidad deben encontrar, cuasiequilibrios altamente dinámicos y transitorios, del núcleo de los requerimientos funcionales de las demandas y las capacidades estructurales de la oferta. Como resultado de esta reflexión se propone que las decisiones instrumentadas a partir de estas capacidades, pueden aceptarse dentro de un rango amplio de estrategias evolutivas eficientes, en un extenso espectro que va desde la adición cercana a los enfoques económicos ortodoxos hasta los actuales de innovación.

\section{PALABRAS CLAVE}

Condiciones para la innovación, cultura organizacional, sostenibilidad.

\section{CLASIFICACIÓN JEL}

L25, M21, O31.

\section{CONTENIDO}

Introducción; 1. Perspectiva evolutiva de la empresa; 2. Complejidad y empresas 3. Condiciones de la innovación: mercado y empresas; 4. Consideraciones finales. Bibliografía.

* $\quad$ Artículo de reflexión elaborado en el marco del proyecto de investigación "Mejoramiento de las capacidades de innovación de las empresas de la región Caribe colombiana", financiado por la Universidad Autónoma del Caribe y la Universidad Simón Bolívar. El proyecto e ejecutó en el período de febrero de 2012 octubre de 2013.

** Sociólogo, Universidad Nacional Autónoma de México, México. Magíster en Economía y Política internacional, Centro de Investigación y Docencia Económicas (CIDE), México. Doctor en Estudios Latinoamericanos, Universidad Nacional Autónoma de México, México. Docente investigador, Facultad de Ciencias Administrativas, Económicas y Contables, Universidad Autónoma del Caribe, Barranquilla, Colombia. Correo electrónico: nestor.sanabria@uautonoma.edu.co.

**** Magíster en Dirección y Organización de Empresas, Universidad Rey Juan Carlos, Madrid, España. Doctor en Dirección y Organización de Empresas, Universidad Autónoma de Madrid, Madrid, España. Docente investigador, Universidad Externado de Colombia. Correo electrónico: julioc.acosta@uexternado.edu.co.

**** Economista, Universidad del Atlántico, Barranquilla, Colombia. Especialista en Estadística Aplicada, Universidad del Norte Barranquilla, Colombia. Doctor en Ciencias Sociales, Universidad del Norte, Barranquilla, Colombia. Docente investigador Facultad de Ciencias Administrativas, Económicas y Contables, Universidad Autónoma del Caribe, Barranquilla, Colombia. Correo electrónico: gustavo.rodriguez51@uac.edu.co. 


\section{Conditions for innovation, organizational culture and sustainability ABSTRACT}

Organizations can be assumed as the result of historic needs found in a certain environment and in the evolutionary process of social systems, hence, their sustainability depends on the capacity of understanding their own complexities. This paper proposes that the provision of conditions for innovation, as an expression of organizational structure, is an option that ensures sustainability and requires a non-uniform non-predictable process, meaning by this a complex process. The analysis suggests that in order for companies to ensure their sustainability, they must find some sort of highly dynamic and transitory equilibrium for the core of the functional requirements from the demand side and the structural capacities for the supply. As a result, this reflection proposes that those decisions instrumented parting from these capacities, can be accepted within a broad range of efficient evolutionary strategies within a broad spectrum that goes from following orthodox economic methods as well as current innovation ones.

\section{KEY WORDS}

Conditions for innovation, organizational culture, sustainability.

\section{JEL CLASSIFICATION:}

L25, M21, O31, P16

\section{CONTENT}

Introduction; 1. Evolutive perspective of the company; 2. Complexity and companies 3. Innovation conditions: Marketing and companies; 4 . Final considerations; Bibliography.

\section{CONDIÇÕES PARA A INOVAÇÃO, CULTURA ORGANIZACIONAL E A SUSTENTABILIDADE \\ RESUMO}

As organizações podem assumir como o resultado das necessidades histórica do entorno e dos sistemas de segurança social em seu processo evolutivo, e a sua sustentabilidade depende da capacidade de compreender a sua própria complexidade. Este artigo propõe que a dotação de condições para a inovação, como uma expressão da cultura organizacional, é uma opção garante da sustentabilidade e requer um processo não-uniforme, nem previsível, ou seja, um processo complexo. As reflexões sugerem que as empresas, de modo a garantir a sua sustentabilidade deve encontrar, quase equilíbrios altamente dinâmico e transitório, do núcleo dos requisitos funcionais de demandas e recursos estruturais da oferta. Como resultado desta reflexão, propõe-se que as decisões implementadas por essas capacidades, podem ser aceitos dentro de uma vasta gama de eficientes estratégias evolutivas, em um amplo espectro que vai desde a adição próximas aos enfoques económicos ortodoxas até os atuais de inovação.

\section{PALAVRAS-CHAVE}

Condições para a inovação, cultura organizacional, sustentabilidade

\section{CLASSIFICAÇÃO JEL}

L25, M21, O31, P16

\section{CONTEÚDO}

Introdução; 1. Perspectiva evolutiva da empresa; 2. Complexidade e empresas 3. As condições da inovação: mercado e empresas; 4. Considerações finais; Bibliografia. 


\section{INTRODUCCIÓN}

En este artículo se plantea que la innovación requiere de condiciones, a partir de la generación de una cultura organizacional apropiada. Para llegar a esas condiciones se debe entender que el proceso innovador no es uniforme y, en general, no acepta claramente los resultados estadísticos lineales y su búsqueda de predicción. La razón es que estos procesos para garantizar su sostenibilidad' deben representar los requerimientos funcionales de la demanda y las capacidades estructurales de la oferta, lo cual simula un proceso evolutivo. Así, las decisiones pueden aceptarse dentro de un rango amplio de estrategias evolutivas eficientes, en un extenso espectro que va desde la adición cercana a los esquemas lineales expresada por la optimización neoclásica basada en presupuestos de racionalidad hasta los linderos de los riesgos en la frontera de lo legítimo que es una de las fuentes de la innovación.

Esta reflexión indaga sobre los aspectos relacionados con los procesos evolutivos de la empresa y su desempeño en la construcción social (Luhmann, 1991 y 2007). En este sentido, el problema derivado de las estrategias de sostenibilidad puede entenderse como una hipótesis y, por tanto, es algo abstracto y en proceso de verificación. Esta hipótesis está teóricamente cercana a los postulados de Schumpeter (1963) sobre la destrucción creativa.

En ambas hipótesis se establece que la función desempeñada por las organizaciones, como instrumento de construcción de desequilibrios (Alchian y Demsetz, 1972), de ampliación del mercado, y de la generación de acumulación económica y social no se resolvió.

Posteriormente y a partir de las nuevas propuestas basadas en servicios, se establecerán nuevos y renovados procesos de competencia, contrarios a las lógicas de uniformidad que subyacen en los anteriores modelos explicativos.

Así, ante las nuevas demandas de soluciones, generadas desde el incremento de complejidad en el sistema-entorno y la imposibilidad de resolverlas con las estructuras empresariales anteriores, se formaron nuevas organizaciones. Esto implicará el reconocimiento de singularidades y anomalías como un hecho fundador de la construcción social (Thom 1972), contrario a los esquemas basados en regularidades. Se puede advertir que la evolución de la organización puede ser comprendida desde la explicación crítica de las fases que conectan la acumulación en un momento y los puntos con estabilidad posteriores que se consoliden como propuestas de cambio.

1 El concepto de sostenibilidad hace referencia a las decisiones que en su capacidad de interpretar el entorno y adaptarse hace que las empresas perduren en horizontes de largo plazo 
A partir de estas realidades, se pueden asumir y explicar las nuevas propuestas evolutivas de las empresas basadas en conocimiento, tecnología e innovación sobre la base de comprender y explotar, producir y reproducir riesgos e incertidumbres. En este sentido las acciones que se consolidan como cambios evolutivos pueden ser entendidas como puntos con estabilidad en el sentido de Lyapunov (citado en Khalil, 1997).

Las consideraciones finales de este artículo se pueden resumir en el sentido de reconocer la existencia de tres formas empresariales básicas y, con variantes regionales o de estilo; todas ellas cubren el espacio social económico administrativo actual. Unas construyen su estrategia de sostenibilidad a partir de escenarios de cooperación entre formas similares. La segunda forma empresarial propia de la propuesta industrial fincó su estrategia de expansión en el establecimiento de reglas obligatorias y voluntarias que generarían una pretensión de uniformidad. Finalmente, con los modernos diseños de innovación y las nuevas tecnologías, la tercera forma apunta a la búsqueda de diferencias con una oferta ampliada a partir de los mercados globales y la comunicación en línea.

En la estructura de esta reflexión se presenta una perspectiva evolutiva del significado de la empresa. Seguidamente, se dan a conocer las teorías sociales diacrónicas de la empresa que responden a los niveles de incremento de la complejidad. Luego, se analiza la relación mercado y empresa desde una aproximación a la teoría de juegos para explicar los riesgos de la innovación y los escenarios de sostenibilidad a partir de estrategias evolutivas eficientes (Sanabria, Chávez y Cantillo, 2012).

\section{PERSPECTIVA EVOLUTIVA DE LA EMPRESA}

De acuerdo con Luhmann (1991), los procesos evolutivos de las empresas son el resultado de los diferentes momentos históricos de los sistemas sociales y de sus entornos. En este sentido, es valioso emprender este análisis con una revisión de su significado. Para ello se puede aceptar que en el transcurso histórico se han generado tres tipos básicos de empresa como se puede entender a partir de lo propuesto por Foucault (2007)2:

a) La primera forma de empresa es el resultado de la construcción mítica y mágica del mundo antiguo y se basa en la cooperación3 en razón, principalmente, de

2 Foucault (2007) plantea razones de gobierno asociadas a lógicas estratégicas históricamente delimitadas, que abarcan la construcción social. Así una razón de mayor gobierno fundamentará la lógica del pensamiento antiguo que dará paso a menor gobierno que implica las maneras que se consolidarán con la ilustración y corresponden a las sociedades industriales. En este sentido se asumen los tipos básicos de empresa.

3 Aunque en muchos casos implica una relación violenta o subyugante, no por ello deja de ser cooperación, como lo presenta Heguel (1966) 
las necesidades de sobrevivencia física. En este período de la construcción civilizatoria descrito por Elías (1987, p. 447 y ss.), se diseñan dos estructuras organizativas, una con alcance local en razón de la incapacidad de ir más allá del entorno cercano y, otra, soporte de las grandes aventuras organizadas por los líderes del momento. Una característica de este modo de construir sociedad, a partir de las empresas, es el lento proceso de acumulación. Este tipo de empresas dejará de liderar la construcción de sociedad, debido al incremento del tamaño de la población, la insuficiencia de la institucionalidad reguladora de los conflictos, el ineficaz ejercicio del poder y las nuevas e incrementales demandas de productividad. En esta realidad se produce una transmutación doble del poder corporativo y del Estado. El primero, más orientado al intercambio comercial sobre la base de las riquezas y productos generados en lo que después se denominará sector primario, y el segundo se vuelve más político. Perdurarán los pequeños y micro-negocios como saldo del periodo, con una cultura que, sin mucha modificación, llegará hasta nuestros días.

b) La segunda forma de empresa, cuya expresión emblemática es la estructura fabril, desarrolló una cultura que también llega hasta nuestros días, tales como las empresas manufactureras con un amplio sector servicios en calidad de subsidiario. El fundamento de esta perspectiva se puede sintetizar en 1. El presupuesto de racionalidad, incluida la limitada (Simon, 1957), cuyo resultado se expresa en los modelos e intenciones de maximización de utilidades u optimización neoclásica; 2. se implementará una institucionalidad ex ante. Esta aparecerá como condición necesaria y suficiente para la valoración de las acciones sociales; y, 3. un proceso de estandarización a gran escala a partir de los inventos, mejora de procesos o resultado del avance tecnológico y una demanda insatisfecha, con pocas posibilidades de conexión, subordinada a las posibilidades de las ofertas locales.

Así, en la versión más ortodoxa de esta forma de empresa y basada en la optimización de precios, se ubicó como función principal la captura de rentas como intención prioritaria del empresariado y; en consecuencia, la ampliación del mercado y la satisfacción de necesidades de los consumidores. Como resultado, se hizo más complejo el sistema y su entorno, y se redefinió la acumulación en la estructura social: desde la agregación de valor a partir del trabajo no cualificado de los individuos, como un factor asociado a los procesos en el sector primario, y los procesos artesanales, a un tema más complejo compuesto por dos factores: el capital y el trabajo. Aunque inicialmente se supondrá el cambio tecnológico como poco relevante, con el tiempo se transformará, por virtud de la evolución de los factores de producción, en el elemento de mayor impor- 
tancia. Se pretenderá también, en lo social, uniformar los comportamientos y preferencias mediante la explicación de las normalidades estadísticas de los resultados empresariales.

Uno de los elementos significativos es la existencia de la estructura de precios como condición ex-ante. En los equilibrios de la oferta y la demanda, se encuentra latente el supuesto de la competencia como condición para la operación eficiente de los mercados. Este enfoque se resume bajo los siguientes supuestos: a) un consumidor racional que a partir de gustos uniformes consulta los precios y toma decisiones, b) una oferta que ajusta sus precios con modificaciones en el tamaño de la escala o los rendimientos decrecientes y procura productos igualmente uniformes a fin de satisfacer la comparación racional de los consumidores. Este enfoque toma como unidad explicativa a una empresa y consumidores medios, que priorizan sus ingresos de corto plazo.

En esta forma ideal de empresa, el saber administrativo camina de la mano de la economía y sus grandes teorías desde varios enfoques y escuelas. Este enfoque tiene como contrapartida: autores como Williamson $(1991,1996)$ quien considera la acción del empresario; los planteamientos de la empresa media de Marshall descritos por Hague (1958); los aportes a la relación empresaempresarios y las oportunidades de negocios presentados por Shackle (1986); la teoría del emprendimiento de Kirzner (1998) y Schumpeter (1997); los costos de transacción formulados por Coase (1937); la teoría de la racionalidad limitada de Simon, (1991); el tema de los incentivos y recompensas en Alchian y Demzets (1972); las teorías del crecimiento de la organización según Arrow (1962 y 1986) y la teoría de la agencia de Jensen y Mecklin (1976), entre otros.

La existencia de esta propuesta de nueva organización se desarrolló en casi dos siglos a partir de invenciones como el motor de vapor que permitió incrementar el mercado y la movilidad humana, lo cual, de acuerdo con Acemoglu y Robinson (2012), generó nuevas instituciones que van desde perspectivas sindicales hasta la actual Organización Mundial del Comercio. Sin embargo, aun cuando esta nueva forma empresarial tiene una alta dinámica, no implicará la eliminación de la primera forma de empresa.

Desde esta perspectiva, las empresas marcharán al ritmo de lo nuevo, y esto lo establece la manufactura. Por tanto, lo más dinámico no implica destrucción sino la creación de una cultura de soporte que se expresa en las empresas en su papel de instancia de reducción de los niveles de complejidad alcanzados. Así son explicables no solo la uniformidad a partir de la estandarización, expresadas en normas técnicas, comerciales o del derecho civil, sino también es entendible 
el papel complementario de la primera forma de empresa.

c) La tercera forma de empresa asume las siguientes consideraciones:

1 Los acumulados del producto mundial muestran predominio del sector servicios considerados estos de manera ampliada. En estos se comprenden los clásicos servicios de la fase industrial, el comercio y las nuevas versiones desarrolladas a partir de los avances tecnológicos que se asumen como economía del conocimiento.

2 Las tecnologías de información y de las comunicaciones han permitido una acción económico-social mucho más eficaz y eficiente de los emprendedores.

3 A diferencia de la fase industrial y su característica principal de rendimientos, tecnología y tamaño de la escala de producción, los resultados empresariales de esta tercera forma pueden ser mayores en la medida en que la base de su estrategia se funda en la endogenización de la información proveniente de las necesidades funcionales de la demanda. Ello implica el redimensionamiento de riesgos e incertidumbres en el ejercicio empresarial, en el sentido de la captura de información y su solución en función de la expansión de las empresas y su prestigio. Se generan entonces capacidades competitivas de orden superior (Porter, 2007).

Estas consideraciones pueden ser entendidas como una estrategia evolutiva eficiente (Sanabria, Chávez y Cantillo, 2012), que se desarrollan a partir del planteamiento de la estrategia evolutiva estable o de equilibrios (Nash, 1950), y la capacidad de adaptación.

\section{COMPLEJIDAD Y EMPRESAS}

Uno de los problemas para explicar las empresas, presentadas en las dos primeras formas, es la incorporación del tiempo lineal continuo de la geometría euclidiana. Esto implica en el ámbito epistemológico la reversibilidad subyacente en la mecánica clásica. Esta dificultad es clara al hacer más complejo el entramado social que fundamenta la existencia de las nuevas propuestas de empresas complejas. En la nueva perspectiva se asumirá que el comportamiento social de las personas es consecuencia de múltiples interacciones (resultado de decisiones) e iteraciones (la generación de culturas o construcción/reconstrucción de escenarios) con diferentes cualidades, intensidades y grado de pertinencia y relevancia.

La manera como se toman las decisiones se refleja en los procesos de la acumulación social, a partir de las empresas, y se puede expresar como puntos con 
estabilidad $^{4}$, sin que pueda predicarse continuidad. Es decir, que a una decisión puede no seguir otra de similares características en razón de que el entorno es cambiante y como resultado del azar. Se denota con ello que la pretensión de uniformidad propia de la cultura fabril y su propuesta de estandarización, expresada en el argot económico como optimización a través de precios homogéneos, si bien construyó sociedad e implementó una estructura institucional de gran valor, llegó a una condición caótica por el propio incremento de complejidad a partir de las nuevas tecnologías de información y comunicaciones, la existencia de una renovada competencia y las múltiples propuestas consolidadas como resultado de la ampliación e interconexión de los mercados internacionales.

Estas circunstancias en las cuales se rebasa la dominancia de lo propuesto en la segunda forma de empresa y el reconocimiento de un nuevo fenómeno emergente permite identificar la tercera forma de empresa. Esta tiene su gran diferencia en relación con las dos primeras, en que tiene su norte no es la estandarización, sino la búsqueda de auto-reconocimiento, en sentido institucional, y segmentación desde un enfoque cultural de los consumidores, a partir de comprender que los puntos con estabilidad o empresas sostenibles no se generan exclusivamente a partir de las capacidades estructurales de la oferta sino principalmente desde los requerimientos funcionales de la demanda.

Una posible solución para algunos casos de empresas, por ejemplo, la producción de commodities en clúster con regular o baja intensidad tecnológica, no implica el abandono estricto de la optimización de precios, sino la redefinición de su variable precio neoclásico, en el sentido del equilibrio, a precio hedónico como diferenciador.

Lo anterior es pertinente para los procesos definidos como innovación radical por Freeman y Soete (1997) cuya característica fundamental es ser productos nuevos y con un ciclo de vida corto en virtud del acelerado cambio tecnológico. También para los procesos de innovación organizacional o innovación social, siempre que generen o modifiquen reglas sociales. Este aspecto es importante en tanto la diferenciación, no solo se debe a un producto con tecnología avanzada, sino que se puede producir sobre un producto estandarizado, como el calzado chino y el cambio en la conducta de los consumidores, apreciable a partir de la emergencia de un tamaño reducido en su escala de producción.

De esta manera, el problema de las empresas actuales no es solo el derivado de la reducción del tamaño de la escala factorial e incrementar los rendimientos;

4 Las organizaciones se pueden considerar puntos con estabilidad en la medida en que cumplen las condiciones de estabilidad de Lyapunov, de estabilidad asintótica y de estabilidad exponencial, lo cual puede traducirse como pertinencia, relevancia y perdurabilidad socialmente considerada. 
también hace referencia al entorno entendido como demanda, dentro de lo que se ha denominado requerimientos funcionales. Es decir, la búsqueda de diferenciación incluye en esta estrategia la existencia de políticas públicas que permitan la reducción de complejidad por las empresas y, como consecuencia, un nuevo liderazgo que implica construcción social y no solo maximización de recursos escasos, como sostiene Robbins (1944).

En este sentido, Teece, Pisano y Shuen (1997) en el enfoque de capacidades dinámicas establecen que estas, al estar constituidas por la habilidad para renovar competencias, mejoran la capacidad de su aplicación y de la interpretación del entorno, a partir de una dirección estratégica cuya pretensión debe ser la obtención de ventaja competitiva. Según Porter y Kramer (2011, p. 34):

La solución está en el principio del valor compartido, que involucra crear valor económico de una manera que también cree valor para la sociedad al abordar sus necesidades y desafíos. Las empresas deben reconectar su éxito de negocios con el progreso social. El valor compartido no es responsabilidad social ni filantropía y ni siquiera sustentabilidad, sino una nueva forma de éxito económico. No está en el margen de lo que hacen las empresas, sino en el centro. Creemos que puede iniciar la próxima gran transformación en el pensamiento de negocios.

Esto implica el desarrollo de nuevos productos y mercados, re-conceptualización de la productividad en la cadena de agregación de valor de las empresas, el establecimiento de asociaciones o clústeres, nuevas políticas y la implementación de responsabilidad social empresarial.

Se puede afirmar, en referencia a las empresas complejas, que en cualquiera de sus formas, su nuevo paradigma requiere talento humano creativo y de mayor costo. Esto es posible si se asumen cuatro hechos relevantes: 1 . Se requiere de creatividad y suficiente capacidad financiera; 2 . Se requiere de suficientes propuestas a fin de que los consumidores puedan discriminar cuál de ellas los identifica para ser implementada; 3. Se requieren territorios innovadores si se desea la sostenibilidad del proceso, y, 4. Un impulso decidido al emprendimiento e intraemprendimiento.

\section{Enfoque adaptativo de las empresas}

Según Rodríguez (2007), la multiplicidad de posibles resultados empresariales es consecuencia de un número considerable y diferenciado de intentos de cambio y adaptación. Los cambios hacen referencia al tránsito que, desde las capacidades estructurales de la oferta, presentan los resultados de las organizaciones complejas ${ }^{5}$,

5 Se hace referencia a las tres formas de empresas descritas en el epígrafe anterior, actuando en escenarios de cooperación y de competencia en dependencia de las formas empresariales, la especificidad de los activos, el nivel de integración y asociación, las capacidades de generar innovación; en fin, en todos aquellos aspectos que hacen que cada unidad sea singular. 
con significativa posibilidad de error debido al alto volumen de información. Esto hace que hechos y acciones se puedan considerar dentro de la categoría de improbables (Luhmann, 2007). Su tránsito posterior a desarrollo y ampliación de mercado irá de lo improbable a lo probable ${ }^{6}$.

Desde la perspectiva funcionalista, las empresas, como organizaciones complejas, reconocen la existencia de entorno y sistema, dotando a este último de la capacidad de definir el cómo de la reducción de complejidad a través de datos; sin embargo, en el entorno, las relaciones a partir de las cuales se construyen los datos no son uniformes, homogéneas, ni parsimoniosas. Estas responden al azar como resultado de las múltiples variables y actores, las interrelaciones entre ellos y la dinámica del todo y de los subconjuntos y, con ello, la dificultad de estabilizar riesgo e incertidumbre.

Esta perspectiva asume la existencia de múltiples formas de empresa, cercana a los planteamientos de Spencer-Brown (1969) ${ }^{7}$ basado en distinciones, en contraposición a los planteamientos ortodoxos de la economía, basados en las identidades. Así, entorno y sistema pueden considerarse reflejo uno del otro, ya que el primero es resultado de interacciones e iteraciones y, el segundo, aparece como captaciones de normas y reglas que transformadas en conceptos, variables y datos permiten expresar, de manera simplificada, las representaciones de formas empresariales.

En este punto es importante señalar que en la literatura base de la teoría de las implementaciones (Hurwicz, 1987; Jackson y Srivastava, 1996; Monsalve, 1999), se puede admitir que las captaciones de la realidad y lo construido como abstracción sistémica implica, además del error resultado del ejercicio de observación parcial de la realidad, los problemas de información incompleta en el sentido de los mercados en tiempo presente, así como los sesgos de las actuaciones de los agentes en momentos futuros. Lo anterior dota de dinámica evolutiva a las empresas según Nelson y Winter (1982), en tanto que ellas se reconozcan no solo como operadoras de las relaciones entre oferentes y demandantes, sino que las precedan normas y rutinas

6 La diferencia con las formas propias de la organización industrial se puede pensar como resultado, en esta última, de los modelos explicativos desde las posibilidades estructurales de la oferta, en tanto que el tercer tipo, como se señaló atrás, el problema apunta a resolverse desde los requerimientos funcionales de la demanda. La literatura sobre organización industrial es abundante. Entre otros: Tirole y Matutes (1990); Mendez y Caravaca (1996).

7 Con este autor se puede interrogar: qué es una organización y para qué sirve. En Law of Form, SpencerBrown plantea que los objetos (cosas, organizaciones y todo aquello que puede ser descrito) no se definen por sus identidades sino por sus distinciones, las cuales son puestas en el objeto por el observador, de manera similar a como se hace con la mecánica cuántica. Por tanto, el observador lo que hace es construir mediante funciones, alegorías o categorías al objeto y, de esta manera, lo que construye un observador solo incluye lo observado, dejando por fuera una gran posibilidad de nuevas distinciones que pueden redefinir el objeto por un nuevo observador, al incorporar cosas que se habían quedado por fuera. 
y, además, busquen adaptar y adoptar nuevas normas que expresen una renovada funcionalidad. A partir de estas normas se construyen nuevas rutinas que hacen del ejercicio competitivo y de las estrategias de sostenibilidad un círculo virtuoso de destrucción creativa como lo formulara Schumpeter (1963).

Así, la diferenciación entre entorno y sistema se da solo en la forma, en razón a sus límites y que uno es continente del otro. Por tanto, es el consumidor quien justifica el cambio de las formas a través de las dudas y las indagaciones, construyendo la necesidad de las estructuras empresariales y su papel de reductoras de la complejidad existente.

En este orden de ideas, son las actuales formas de comunicación, en todos los sentidos y con múltiples contenidos a partir de las tecnologías de información, el problema central a dilucidar. Esto no deja en un estado de minusvalía a los productos, sino que los dota de trascendencia en tanto que dejan de ser sujetos pasivos e ingresan con identidad propia dentro de los imaginarios económico-sociales, a través de sus representaciones en la estructura de comunicación. Esta situación refleja las capacidades de distinguir los fondos y trasfondos de los enunciados o captaciones de la realidad y su resultado presentado, y cómo las capacidades e identidades que posibilitan los datos establecen las funciones y, a partir de ellas, las estructuras con los que se formalizarán los límites difusos de las empresas.

Por tanto, lo estratégico de las empresas adaptativas actuales no es su estructura, expresada como manuales y procedimientos estandarizados, sino su capacidad de comprender y transmitir hechos y objetos emergentes, lo cual corresponde a la generación de capacidad dinámica, como explica Teece (2009).

De esta manera la explicación de las empresas adaptativas se acercaría a la lógica expresada por Becker y Murphy (2000) en fad, fashion and norm. Por tanto, puede ser aceptable asimilar las empresas adaptativas como una interface entorno-sistema. Para ello puede ser necesario aceptar las consideraciones matemáticas de los puntos con estabilidad y los planteamiento de Prigogine y Stenger (1984) y Prigogine (2008) en relación con las estructuras disipativas ${ }^{8}$.

El esquema planteado no ignora las estructuras de los negocios tradicionales o de las dos primeras forma de empresas presentadas en el epígrafe anterior; estas participan en la construcción evolutiva a partir de esquemas de cooperación.

8 Se puede hablar de estructuras disipativas cuando la tendencia evolutiva es hacia el desorden, lo cual puede implicar que las empresas tienen dos claras opciones: o se adaptan al entorno a través de la reducción de complejidades y, con ello, tomar decisiones que garanticen su perdurabilidad, o como tendencia pueden marchar a la extensión. Esto, como se verá adelante, requiere de la no reversibilidad, como formulan estos autores. 
Con base en lo anterior, se puede argumentar que la empresa es un producto social diacrónico que responde a los niveles de incremento de adaptación y complejidad, cuya función es reducir el riesgo e incertidumbre para transformar el entorno. En este sentido, su sostenibilidad refleja su capacidad interpretativa a partir de su estructura estratégica adaptativa, desarrollada como juego de cooperación y competitividad en sus variantes de precios o creatividad.

\section{CONDICIONES DE LA INNOVACIÓN: MERCADO Y EMPRESAS}

En los epígrafes anteriores se describieron tres formas de empresas. De esto se puede desprender que el juego de las empresas corresponde a encontrar la estructura más eficaz en el procesamiento de información, sujeto a las pretensiones de medio y largo plazo establecidos en su ordenamiento estratégico. Este planteamiento permitirá proponer a partir de las captaciones de la realidad, explicaciones e interpretaciones plausibles, transformarlas en conocimiento útil y orientar cambios en el entorno (Sharma y Chrisman, 1999).

Por su parte, el ordenamiento estratégico y su gestión, en los límites aquí presentados, corresponde al puente explicativo que uniría sistema y entorno, y cuyo resultado debe traducirse en mayor creación de valor y consolidación de ventajas competitivas (Madhavan y Grover, 1998; Subramaniam y Youndt, 2005). Es de resaltar cómo a la primera forma de empresa se pueden asociar las ventajas comparativas; a la segunda forma, las ventajas competitivas, y a la tercera forma, las ventajas competitivas de orden superior (Porter y Kramer, 2011).

Ahora bien, la creación de valor y la obtención de ventaja competitiva pueden entenderse como una expresión no lineal y cercana a la geometría fractal (Mandelbrot, 1997). Esto implicaría capacidades de acumulación social que expresan los cambios en el entorno del sistema y que son el fundamento de la responsabilidad social empresarial. Se puede entender entonces que los puntos con estabilidad generados por vía de la innovación, basados en culturas, corresponden a cambios que tienen la posibilidad de ser ciertos en la medida en que sean muchas las propuestas, y que la consolidación de una(s) de estas expresa(n) la evolución social, ya que se puede suponer de ella(s) la mejor capacidad de adaptación y, por tanto, de sostenibilidad.

En el supuesto que existe una cultura de innovación como resultado específico de la construcción de la tercera forma de empresa, entendida esta como proceso evolutivo civilizatorio (Elías, 1987), se pueden establecer diferenciaciones, a través de las formas empresariales emergentes, a condición de que el proceso innovador 
genere ampliación de mercado y reconfiguración de la sociedad con cambios en las distinciones. ${ }^{9}$

Se puede suponer también que al menos uno de los valores asociados a la matriz que expresa a la sociedad es un valor propio asimilable a la probabilidad de construcción-consolidación de nuevos puntos con estabilidad. Ahora bien, la no uniformidad del mercado implicaría que pueden existir múltiples puntos con estabilidad, tantos como el propio proceso evolutivo haga plausibles. Como el evento innovador depende de las condiciones del entorno, en los cuales también participa la dinámica interna de las empresas, en especial la gerencia, se puede asumir, para expresar el problema de mercados y empresas, que los esfuerzos innovadores, una vez abandonan el limbo de la improbabilidad como se presentó antes, se explican como la potencialidad de construcción de ventajas competitivas de orden superior. Con ello se podría contribuir a comprender, desde la perspectiva evolutiva, los ciclos de los mercados y su capacidad de destrucción creativa.

Los puntos con estabilidad, en consecuencia, se pueden asumir como constitutivos de una metáfora que no sigue patrones estables en el corto y medio plazo, por el incremento de entropía en virtud de las tecnologías de información. Estos comportamientos caóticos están ahí como resultado de la existencia de la innovación, como expresión de esa tercera forma de empresa. Se puede asumir que la dinámica implícita del entorno de la empresas expresa las posibilidades conservativas del sistema; por tanto, y en el supuesto de la existencia de la cultura innovadora, en alguna región del mapa de mercado, se generará un nuevo punto con estabilidad que cumpliría con que el límite de la función de distribución de los eventos innovadores tienda a uno cuando la cantidad de apuestas innovadoras sean altas.

De esta forma, la emergencia de eventos y ajustes evolutivos hacia el medio y largo plazo podría implicar la existencia de entornos inestables en las condiciones iniciales y ello permitiría asumir al mercado en permanentes condiciones de inestabilidad, lo cual es evidente si se aceptan los escenarios de sostenibilidad, como en la evolución de las especies planteada por Darwin: el que mejor se adapte a sus propios entornos o capte de mejor manera la realidad como se ha planteado podrá garantizar sostenerse, pero esto solo es temporal en virtud de la competencia.

Lo formulado implica las no linealidades del mercado y la no existencia de una única opción, por estar compuestas por múltiples apuestas expresadas como intereses y adscripciones culturales, resultado de la capacidad de procesamiento de información en el sentido de la posibilidad de captación de información. Se puede

9 Este concepto se presenta en sentido similar a lo expuesto en Bordieu (2002, 35 y ss.) 
asumir, entonces, que el mercado se comporta como una integral de caminos en donde se agregan las diferentes opciones funcionales expresadas. Esto acepta la existencia de múltiples puntos con estabilidad, que en su sumatoria compleja, hoy se asimilan a los mercados más dinámicos.

De esta manera, también es posible asumir que la existencia de trayectorias permite establecer diferencias de fase y explica en términos evolutivos por qué una opción local es más probable que otra en uno o más escenarios de mercado. También puede aproximar a una explicación de la existencia de complementariedades, entre micro negocios y grandes superficies comercializadoras y el que las grandes crecen más, en tanto que les quiten márgenes de mercado a los micros o pequeños negocios.

Se pueden asumir, en consecuencia, dos magnitudes en resultado del ejercicio competitivo de mercado en relación con las empresas. Por un lado, el precio como una de sus probabilidades, y por otro, las fases-caminos como expresiones de deriva aleatoria que identifican de mejor manera a la innovación ${ }^{10}$. Con ello, se puede reconocer que la ampliación del mercado correspondiente al fin último de la competitividad, es resultado de la suma de la contribución de todas las interacciones e iteraciones existentes a partir de las empresas y el papel de agentes y agencias. Es decir, de la operación simultánea de todos los tipos ideales descritos, a condición de que la tercera forma de empresa establezca como identidad su sostenibilidad.

Las diferencias existentes entre los puntos con estabilidad van a permitir que en la integral de caminos se descuenten las capacidades que sean contrarias ${ }^{11}$, no reducible su complejidad (por ejemplo, inventos sin desarrollo de mercado), no competitivas o no participantes de juegos cooperativos. Así, la esencia del mercado: el juego entre competencia y/o cooperación (Nalebuff y Branderburguer, 1996), implicará un proceso de juegos revolucionarios en los cuales no existe ninguna opción predeterminada.

Los resultados aleatorios se puede ejemplificar como: es posible que una gran superficie comercial asuma una función del micro-negocio y, en el saldo final, se pueden asumir como jugadas inteligentes de estos últimos, el generar espacios funcionales a la gran superficie y la manera evolutiva de mantenerse y sostenerse, según Sanabria, Chávez y Cantillo (2012). De igual consideración, en relación con lo expresado por la termodinámica, las políticas de competitividad supondrían la

10 Existen otras opciones para explicar los comportamientos no lineales y con raíz unitaria, pero para los intereses de este artículo solo se asimilara la deriva aleatoria.

11 En el sentido que sea absorbida a través de funcionalidades copadas por productos nuevos o vía renta con disminución del tamaño de la escala, por ejemplo. 
capacidad de determinar el más probable camino de mínimo esfuerzo de las acciones sociales (productividad-innovación-costo), establecido como la ruta más eficiente en la construcción probabilística de los precios.

Con ello, el problema se acerca a la solución teórica de los precios formulada por Cantillón (1950). De esta manera, el precio es asimilable a un costo de oportunidad (Thornton, 2007, 2009) y contempla las opciones de la competencia. Ello implica que se puede aceptar una aproximación entre sistema y entorno a partir de entender la relación entre organizaciones y mercado, mediante la interpretación desde el mecanismo de las integrales de caminos. Aparecen entonces las variantes de innovación como soluciones eficaces que permiten relacionar las necesidades funcionales de la demanda, expresadas como reglas sociales, y las capacidades de respuesta de la oferta a través de sus empresas.

\section{CONSIDERACIONES FINALES}

Es importante tener en cuenta que hoy las tres formas de empresas descritas coexisten de manera simultánea y todos participan del mercado en la pretensión de garantizar su sostenibilidad. Se puede pensar que las micros y pequeñas empresas, cercanas a la primera forma, han perdurado en razón a que su fuerza pareciera depender de la cantidad de sus unidades. Su juego se asemeja a lo establecido por Akerlof (1976) como una rat race en una aparente estrategia evolutiva estable en el corto plazo (Axelrod, 2006), definida a partir de asumir su tendencia a contar con un número grande de ellas en una estructura de equilibrio de Nash en el cual, la destrucción o la eliminación parcial de un número de sus asociados, aunque importante, no implica su colapso o afectación grave de sus estructuras sociales.

De manera contraria, una falla significativa conducente a la destrucción de una gran unidad de las empresas, de la segunda y tercera forma, puede implicar un conflicto que afecte todo el conjunto social y tocar, incluso, importantes mecanismos de distribución y canales de legitimación. Lo formulado puede contribuir a explicar por qué la acción innovadora se presenta en mayor medida en la estructura grande que en la micro y pequeña empresa, aun cuando estas últimas, de conjunto y en el largo plazo, son más sostenibles, lo cual se puede asumir como una estrategia evolutiva eficiente (Sanabria, Chávez y Cantillo, 2012).

En este sentido, el conflicto derivado de la estrategia de sostenibilidad puede entenderse como una hipótesis y, por tanto, es algo abstracto y en proceso de verificación. En esta perspectiva, los problemas de desequilibrios en las diferentes escalas de acumulación y supervivencia de la primera forma de empresa no fueron 
resueltos, y su intento de resolución dio paso a la construcción de la segunda forma de empresa, y su construcción cultural se registra como la etapa industrialista de la historia. Aunque de mejores fundamentos en gestión y productividad, dará paso, con la emergencia de las tecnologías de información y comunicaciones, a la tercera forma de empresa.

Se puede entender entonces el proceso evolutivo a partir de las respuestas ante las nuevas demanda de soluciones. Estas son el fundamento del incremento de complejidad en el sistema-entorno. Esto implicará el reconocimiento de singularidades y anomalías como un hecho fundador de la construcción social (Thom 1972) como puede predicarse de la tercera forma y contrario a los esquemas basados en regularidades, pero complementario en los escenarios económicos administrativos, en los cuales las necesidades de lo estandarizado siguen siendo un gran mercado y permiten el piso mediante la creatividad de la puesta en escena de las nuevas soluciones.

A partir de estas realidades, se pueden asumir y explicar las nuevas propuestas evolutivas de las empresas basadas en conocimiento, tecnología e innovación sobre la base de comprender y explotar, producir y reproducir riesgos e incertidumbres. En este sentido las acciones que se consolidan como cambios evolutivos pueden ser entendidas como puntos con estabilidad en el sentido de Lyapunov (cfr.: Khalil 1997) en el cual el tiempo, considerado como cambio, tiene un sentido y este es construido por las decisiones empresariales y se constituye en el elemento de mayor dinámica y soporte de la construcción de ventajas competitivas de orden superior.

\section{BIBLIOGRAFÍA}

Acemoglu, D. y J. A. Robinson (2012). Porqué fracasan los países. Barcelona: Deusto, 608p.

Akerlof, G. (1976). The economics of caste, and of the rat race and other woeful tales. En: Quaterly Journal of Economics, Vol. 90, p. 599-617.

Alchian, A. y H. Demsetz (1972). Production, Information Costs, and Economic Organization. En: American Economic Review, Vol. 62, № 5, p. 777-795.

Axelrod, R. (2006). The evolution of cooperation. New York: Basic Books, 24lp.

Arrow, K. J. (1962). The economic implications of learning by doing. En: Collected Papers of Kenneth J. Arrow, Vol. 5, Production and Capital, Harvard University Press, Cambrige, Mass, 496p.

Arrow, K. J. (1986). Rationality of self and others in an economic system. En: Journal of Business, Vol. 59, N 4, p. 385-399. 
Condiciones para la innovación, cultura organizacional y sostenibilidad de las organizaciones

Becker, G. S. y Murphy, A. (2000). Fad, fashion and norm. En: Social Economics, Market Behavior in a Social Environment. Massachusetts: Boston: Harvard University Press, 170p.

Bordieu, P. (2002). La distinción. Criterio y bases sociales del gusto. México: Taurus, 600p.

Cantillon, R. (1950). Ensayo sobre la naturaleza del comercio en general. México: F. C. E., 234p.

Coase, R. H. (1937). The Nature of the Firm. En: Económica, Vol. 4, N. 16, p. 386-405.

Elias, N. (1987). El proceso de la civilización. México: F. C. E., 674p.

Foucault, M. (2007). Nacimiento de la biopolítica. México: F. C. E., $401 p$.

Freeman, C. y L. Soete. (1997). The economics of industrial innovation. Third Edition. Londres: Printer, 470p.

Gadamer, H. G. (1993). Verdad y método. Tomo 1. Salamanca: Ediciones Sígueme, 687p.

Hegel, G. W. F. (1966). Fenomenología del espíritu. México: FCE., 1176p.

Hague, D. C. (1958). Alfred Marshall and the competitive firm. En: The Economic Journal 272, Vol. 68, p. 673-690.

Hurwicz, L. (1987). Incentives aspects of decentralization, p. 1441-1482. En: Arrow, K. y M. Intrilligator. Eds. Handbook of Mathematical Economics, Vol. III. Amsterdam: North Holland, 1486p.

Jackson, O. y S. Srivastava. (1996). A characterization of game theoretic solutions which lead to impossibility theorems. En: Review of Economic Studies, Vol. 63, Nº 1, p. 23-38.

Jensen, M. y W.H. Mecklin. (1976). Theory of the firm: managerial behaviour, agency costs and ownership structure. En: Journal of Financial Economics, Vol. 3 N 4, p. 305-360.

Khalil, H. (1997). Stability. En: Levine, W. The Control Handbook. Boca Ratón, Florida: IEEE, p. 889-897

Kirzner, I. (1998). Competencia y empresarialidad. Madrid: Unión Editorial, 270p.

Luhmann, N. (1991). Sistemas sociales. Lineamientos para una teoría general. México: Alianza I Universidad Iberoamericana, $171 \mathrm{p}$.

Luhmann, N. (2007). La sociedad de la sociedad. México: Universidad Iberoamericana-ITESO/Editorial Anthropos, 1024p.

Madhavan, R. y R. Grover. (1998). From Embedded Knowledge to Embodied Knowledge: New Product Development as Knowledge Management. En: Journal of Marketing, Vol. 62, N 4, p. 1 12.

Mandelbrot, B. (1997). La geometría fractal de la naturaleza. Barcelona: Tusquets Editores, 680p.

Monsalve, S. (1999). Introducción a los conceptos de equilibrio en economía. Bogotá: Unibiblos, 358p. 
Méndez, R. y Caravaca, I. (1996). Organización industrial y territorio. Madrid: Ariel.

Milnor, J. (1985). On the concept of attractor. Communications of Mathematical Physics, Vol. 99, $\mathrm{N}^{\circ}$ 2, p. 177-195.

Nash, J. F. (1950). Non cooperative Games. En línea] Ph. D. Dissertation Princeton. Consultado en julio 15 de 2005, 32p.

Nalebuff, B. y A. Branderburguer. (1996). Coo-petencia. Bogotá: Norma, 432p.

Nelson, R. y Winter, S (1982). An evolutionary theory of economic change. Cambridge: Harvard university press, $440 p$.

Porter, M. (2007). Estrategia competitiva. México: Grupo Editorial Patria.

Porter, M. y Kramer, M. (2011). Creating Shared Value. How to reinvent capitalism -and unleash a wave of innovation and growth. En: Harvard Business Review, enero-febrero, 2011. Consultado en julio 30 de 2013.

Prigogine, I. (2008). Las leyes del caos. Barcelona: Drakontos, 161p.

Prigogine, I. y Stengers, I. (1984) Order out of Chaos: Man's New Dialogue with Nature. New York: Bentam.

Rodriguez, D. (2007): Presentación de la edición en castellano. En Lhumann, N. 2007: La sociedad de la sociedad. México: Herder, 1024p.

Robbins, L. (1944). Ensayo sobre la naturaleza y significación de la ciencia económica. México: FCE, 214p.

Sanabria, N. J., N. Chávez y J. D. Cantillo. (2012). Globalización y evolución empresarial, estudios del sector servicios. En: Universidad y Empresa, Vol. 21, p. 37-63.

Schumpeter, J. A. (1963) Capitalismo, socialismo y democracia. 3. ${ }^{a}$ edición; México: Aguilar. 512p.

Schumpeter, J.A. (1997). Teoría del desenvolvimiento económico. México: FCE, 256p.

Shackle, G. (1986). The Origination of Choice. En: Kirzner, I. editor. Subjectivism, Intelligibility and Economic Understanding. Londres: Macmillan, p. 281-287.

Sharma, P. y J.J. Chrisman. (1999). Toward a reconciliation of the definitional issues in the field of corporate entrepreneurship. Entrepreneurship: Theory and Practice, Vol. 23, p. 11-27.

Simon, H. (1957). Models of man. New York: John Wily and Sons, Inc., 201p.

Simon, H. (1991). "Organizations and Markets." En: Journal of Economic Perspectives 2, Vol. 5, p. 25-44. Spencer-Brown, G. (1969). Law of form. Londres: Allen and Unwin, 141p. 
Subramaniam, M. y M. Youndt. (2005). The Influence of Intellectual Capital on the Types of Innovative Capabilities. En: Acad. Manage Journal, Vol. 48, N³, p. 450-463.

Teece, D. (2009). Dynamic capabilities. Oxford: Oxford University Press, 286p.

Teece, D. J., Pisano, G., y Shuen, A. (1997). Dynamic capabilities and strategic management. En: Strategic Management Journal, Vol. 18, Nº 7, p. 509-533.

Thom, R. (1972). Stability structurally and morphogenesis. Nueva York: Benjamin, 348p.

Thornton, M. (2007). Was Richard Cantillon a Mercantilist? En: Journal of the History of Economic Thought, Vol. 29, p. 417-435.

Thornton, M. (2009). Cantillon and the Invisible Hand. En: Quarterly Journal of Austrian Economics, Vol. 7, p. 27-46.

Tirole, J, y Matutes, C. (1990). La teoría de la organización industrial. Madrid: Ariel, 734p.

Williamson, O. y Winter, S. (Eds.) (1991) The Nature of the Firm: Origins, Evolution, and Development. México: FCE.

Williamson, O. (1996) The Mechanisms of Governance, New York: Oxford University Press. 
